Témoigner Témoigner. Entre histoire et mémoire

Getuigen Revue pluridisciplinaire de la Fondation Auschwitz

$116 \mid 2013$

Voyages mémoriels

\title{
Dix ans de voyages pédagogiques à Auschwitz : bilan critique
}

Ten years of educational visits to Auschwitz: a critical review

Tien jaar pedagogische reizen naar Auschwitz: een kritische balans

Pierre-Jérôme Biscarat

\section{(2) OpenEdition}

Journals

\section{Édition électronique}

URL : http://journals.openedition.org/temoigner/293

DOI : 10.4000/temoigner.293

ISSN : 2506-6390

Éditeur :

Éditions du Centre d'études et de documentation Mémoire d'Auschwitz, Éditions Kimé

Édition imprimée

Date de publication : 1 septembre 2013

Pagination : $33-41$

ISBN : 978-2-84174-643-9

ISSN : 2031-4183

Référence électronique

Pierre-Jérôme Biscarat, « Dix ans de voyages pédagogiques à Auschwitz : bilan critique », Témoigner. Entre histoire et mémoire [En ligne], 116 | 2013, mis en ligne le 01 juin 2015, consulté le 10 décembre 2020. URL : http://journals.openedition.org/temoigner/293 ; DOI : https://doi.org/10.4000/temoigner 293 


\title{
Dix ans de voyages pédagogiques à Auschwitz : bilan critique
}

\author{
PIERRE-JÉRÔME BISCARAT
}

Maison d'Izieu

«Quand je faisais Shoah [...] je me disais toujours, mais au fond, à quoi ça sert [...] les questions que tu poses [...] six millions ont été tués, on sait... Pourquoi vouloir arpenter des lieux vides et puis décider que là était l'entrée du camp... Et en fait, six millions, c'est un résultat, c'est une abstraction et seuls les détails permettent d'accéder à la vérité, à la réalité, à ce que cette abstraction masque et cache [...]. Ça sert à faire en sorte que la vérité soit. Ça sert à l’incarner, vouloir que la vérité soit dite, que la réalité de cette épouvante soit dite, vouloir le comment. Le détail le plus extrême du comment, c'est une loi absolue. »

« Auschwitz ne se visite pas [...] lire seulement n'est pas assez. Il faut voir et savoir, savoir et voir, indissolublement. C'est un travail déchirant. »

Claude Lanzmann ${ }^{1}$

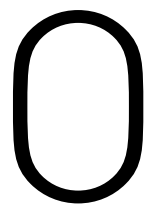

$\mathrm{n}$ me demande de dresser un bilan critique de dix ans de voyages pédagogiques à Auschwitz ${ }^{2}$. Je ne parlerai pas des voyages à Auschwitz pour le grand public, mais des voyages uniquement pédagogiques impliquant des élèves, des enseignants et des institutions mémorielles. Je propose une intervention à la première personne, sans langue de bois. Si mes jugements peuvent paraître parfois trop tranchés, c'est pour mieux susciter le débat. Mes réflexions s'appuient sur plus de dix années de pratiques professionnelles. Je travaille à la Maison d'Izieu où j’anime les ateliers pédagogiques, élabore des programmes

(1) «Entretien avec Jacques Toubiana », émission Bandes à part, Radio France Culture, 14 octobre 2001 ; Claude Lanzmann, « Préface », in Filip Müller, Trois ans dans une chambre à gaz d'Auschwitz, Paris, Pygmalion, 1980, p. 9-17.

(2) En 2011, trois séminaires ont été organisés pour réfléchir sur les voyages pédagogiques : «Voyages d'études et de mémoire à Auschwitz - enjeux contemporains » par le Mémorial de la Shoah et le ministère de la Défense (DMPA), des 24 et 25 mars ; «Voyages pédagogiques sur les lieux de mémoire de la Shoah » par la Fondation pour la Mémoire de la Shoah, à l'ENS Lyon, du 20 au 22 mai ; « Les voyages de mémoire de la Shoah » par le Centre universitaire Jean-François Champollion et l'Association des amitiés judéo-lacaunaises, des 17 et 18 septembre (Jacques et Ygal Fijalkow, Les Élèves face à la Shoah. Lieux, histoire, voyages, Albi, Presses du Centre universitaire Jean-François Champollion, 2013, 297 p.) 
de formations pour les enseignants et poursuis des recherches historiques. Je siège également à la commission « Enseignement » de la Fondation pour la Mémoire de la Shoah. J'organise, depuis 2000, sur le plan technique, historique et pédagogique des voyages d'élèves de plusieurs jours à Cracovie et à Auschwitz. Depuis 2005, je conduis avec l'historien Jean-François Forges, un séminaire annuel d'études pour les enseignants et les professionnels des musées sur « Les traces juives de Cracovie, le camp d'Auschwitz et le crime contre l'humanité » avec la Maison d'Izieu, le Centre d'Histoire de la Résistance et de la Déportation de Lyon et la Chaire lyonnaise des Droits de l'Homme.

J'ai effectué mon premier déplacement en Pologne, en mars 1998, avec quatrevingts étudiants et cinquante lycéens de la région de Lyon, un voyage organisé par l'association antinégationniste Hippocampe ${ }^{3}$ dont j'étais le fondateur. Un périple de cinq jours nous a conduits du camp de concentration du Struthof en Alsace jusqu'au complexe d'Auschwitz en Pologne. Nous étions accompagnés d'historiens et d'anciens déportés. Si je reprends nos déclarations de l'époque :

Ce voyage est né d'un ras-le-bol face aux dérapages racistes, antisémites et négationnistes des enseignants du Front national de l'Université Lyon III. Ce projet, à dimension européenne, entend promouvoir la mémoire de la Résistance et de la Déportation. Il a pour objectifs de provoquer une réflexion active autour du concept de mémoire et de citoyenneté. Ce voyage entend clore une des phases de notre engagement citoyen. Non pas qu'il faille aller à Auschwitz pour être un citoyen accompli, mais ce voyage permet d'illustrer concrètement les valeurs fondamentales de responsabilité, de conscience et de mémoire.

Le voyage en car, de Lyon à Cracovie, a duré plus de trente heures, entre les conditions climatiques et le contrôle des passeports aux frontières. La visite s'est effectuée en une seule journée. Le matin, nous avons rapidement traversé en car le quartier juif de Kazimierz, avant de rejoindre pour l'après-midi le Musée d'Auschwitz. Très peu préparé, j'ai parcouru les espaces historiques d'Auschwitz I et de Birkenau sans pouvoir les comprendre, sans pouvoir les lire. Il a été difficile de se repérer dans le temps, l'espace et les multiples logiques d'Auschwitz. Nous avons été confrontés à quatre problèmes majeurs :

\section{L'immensité du complexe d'Auschwitz}

Il se compose de trois sites principaux : Auschwitz I, Auschwitz II - Birkenau et Auschwitz III - Buna-Monowitz, auxquels se rajoutent les nombreux Kommandos extérieurs, comme Rajsko, Budy ou Bobrek, ainsi que les fosses communes et les multiples gravières, lieux d'exécutions et de travaux mortels. Certains de ces lieux sont associés à de grandes personnalités : Auschwitz III pour Primo Levi ${ }^{4}$, Bobrek

(3) Organisation conjointe avec Mnémosyne, association étudiante de l'Université Lyon II.

(4) «[...] la Buna est désespérément grise et opaque. Cet interminable enchêtrement de fer, de ciment, de boue 
pour Simone Veil5. Nous ne les avons pas vus. Et pourtant, combien d'entre nous n’avaient-ils pas lu « Si c'est un homme » pour se préparer au voyage.

\section{L'imbrication de logiques différentes dans un même lieu}

L'espace historique lié aux structures de mise à mort est imbriqué dans l'espace des parties concentrationnaires, alors qu'il s'agit de deux logiques différentes, de deux mondes bien distincts. Lors des visites, il y a une véritable difficulté pour concilier la succession topographique des lieux et les logiques historiques spécifiques.

\section{La compréhension des mémoires multiples d'Auschwitz}

Elles se télescopent parfois les unes dans les autres dans le même espace mémoriel : mémoire des prisonniers de guerre soviétiques, mémoire juive, mémoire tsigane, mémoire résistante polonaise, mémoire des prisonniers politiques ou militaires d'autres pays occupés et mémoire des « asociaux ». Les nazis réservant à chacune des catégories un sort spécifique et singulier. En plus de ces différentes catégories de victimes, il faudrait prendre en compte les mémoires nationales des pays touchés par la déportation. Par exemple, les Juifs déportés à Auschwitz représentent au moins treize pays : Hongrie, Pologne, France, Pays-Bas, Grèce, Bohême-Moravie, Slovaquie, Belgique, Allemagne et Autriche, Yougoslavie, Italie, Lettonie et Norvège.

\section{Des sites d'exterminations en ruines}

La partie du centre de mise à mort de Birkenau n'est plus qu'un vaste champ de ruines. Les Crématoires II, III, IV et V et les Bunkers I et II sont désormais des sites archéologiques. Les zones des bûchers et les fosses communes sont devenues de vertes prairies et les lieux de dépôt des cendres des victimes, de petites mares. Comment connaître, puis lire ces lieux sans un intense travail de préparation ${ }^{6}$ ?

Finalement, au cours de ce premier voyage, je n'ai rien vu à Auschwitz ; je n'ai rien compris d'Auschwitz. De plus, notre voyage s'est trouvé pris dans l'actualité politique avec les élections régionales. Le 22 mars 1998, le président de la Région Rhône-Alpes, Charles Millon, conservait son siège grâce à un accord avec le Front national. Lémotion était considérable. De Cracovie, nous avons lancé un communiqué

et de fumée est la négation même de la beauté. [...] La Buna est aussi grande qu'une ville. Outre les cadres et les techniciens allemands, quarante mille étrangers y travaillent, et on y parle au total quinze à vingt langues. Tous les étrangers habitent dans les différents Lager qui entourent la Buna. » Primo Levi, Si c'est un homme, Paris, Julliard, 1987, p. 93-94.

(5) « Nous sommes restés à Bobrek à quatre ou cinq kilomètres de Birkenau de juillet 1944 à janvier 1945. [...] Nous sommes arrivés à Bobrek, deux ou trois jours avant mon anniversaire. [...] Le commando comptait environ deux cent cinquante déportés, dont trente-sept femmes. Nous étions répartis entre des tâches diverses en liaison avec les activités de l'usine Siemens qui fabriquait des pièces d'avions dont je n'ai jamais vu une seule, car ma sœur et moi avions été affectées aux éternels travaux de terrassement. » Simone Veil, Une Vie, Paris, Stock, 2007, p. 78-81.

(6) À la fin des années 1990, les indications et les explications de ces sites étaient très sommaires, voire inexistantes. Depuis quelques années, le Musée d'État d'Auschwitz, sous la direction de Piotr Cywinski, a comblé ces lacunes. Un important réseau de panneaux permet de se situer dans les différents sites et de disposer d'une information historique synthétique et rigoureuse. 
de presse dénonçant cet accord. Ce fut un voyage politique et citoyen réussi, mais un échec pour la connaissance historique des lieux. Bref, nous étions dans la catégorie du « voyage symbolique ». Et les démarches symboliques ne s'embarrassent pas de l'approche historique. De cet échec est née ma détermination à retourner en Pologne afin de mieux appréhender ces lieux.

En juin 1999, le Père Patrick Desbois ${ }^{7}$, responsable de l'association Teshouva, Richard Prasquier, président de Yad Vashem France et Geneviève Erramuzpé, directrice de la Maison d'Izieu, m’ont proposé de participer à un voyage d'études de dix jours sur les traces des Juifs polonais et les camps nazis. Nous avons visité Varsovie, Belzec, Majdanek, Sobibor, Cracovie ainsi que Lvov et le camp de Rawa-Ruska en Ukraine. Pour la visite d'Auschwitz, nous étions guidés par l'historien italien Marcello Pezzetti", accompagné de Shlomo Venezia, ancien membre du Sonderkommando'. Nous avons étudié en détail tous les lieux importants des sites d'Auschwitz I et de Birkenau. Marcello Pezzetti appuyait ses propos sur des documents d'archives. Tous les éléments étaient ainsi réunis pour une véritable approche de l'histoire d'Auschwitz : le lieu, le témoin, l'historien et les documents. En juillet 2000, les organisateurs ont renouvelé ce séminaire de manière encore plus approfondie. Ces deux voyages d'études ont été déterminants dans ma formation et ma vocation.

Par la suite, j’ai organisé mes premiers voyages pédagogiques pour le compte de la Maison d'Izieu. Parallèlement, j’ai accompagné d'autres groupes comme simple observateur. J'ai été attentif aux différents voyages, me procurant les programmes et quelques travaux d'élèves. J'ai lu dans la presse le compte rendu heureux ou malheureux de ces voyages qui n’ont cessé de se développer au cours des années 2000. Fort de ces expériences, j’ai pu observer quelques écueils récurrents :

\section{LE TEMPS COURT}

Lavisite en une seule journée, voyage compris, m’apparaît difficilement compatible avec une démarche historique et pédagogique. Pour avoir une idée concrète de ce type de formule, je prendrai pour exemple un voyage d'une journée « Étude et mémoire » à Auschwitz-Birkenau organisé par le Conseil général du Rhône pour cent cinquante collégiens de quinze collèges, accompagnés de leurs professeurs et de survivants :

Le mercredi 13 janvier 2010, nous décollons de Lyon pour Cracovie à $18 \mathrm{~h}$. Nous arrivons à destination à $20 \mathrm{~h} 30$. Le lendemain, jeudi 14 janvier, le réveil est fixé à $5 \mathrm{~h}$ 30, le petit-déjeuner à $6 \mathrm{~h} 15$. Le départ pour le musée d'Auschwitz est à $6 \mathrm{~h} 45$. Le temps du trajet de Cracovie pour le musée d'Auschwitz est de $1 \mathrm{~h} 30$. Après une brève présentation, la parole est donnée à un témoin. Il témoigne dans le car tout en

(7) Patrick Desbois, Porteur de Mémoires, Neuilly-sur-Seine, Michel Lafon, 2007.

(8) Serge Klarsfeld, Marcello Pezzetti, Sabine Zeitoun, L'Album d'Auschwitz, avant-propos de Simone Veil, Romainville, Al Dante/Fondation pour la Mémoire de la Shoah, 2005 ; Marcello Pezzetti, Destinazione Auschwitz, 2 cd-roms et 1 DVD, Milan, Proedi, 2002.

(9) Shlomo Venezia, Béatrice Prasquier, Sonderkommando. Dans l'enfer des chambres à gaz, préface de Simone Veil, Paris, Albin Michel, 2007. 
répondant aux questions des élèves. Nous arrivons à Auschwitz I à 8 h 45 . À l'entrée, chacun des huit groupes est pris en charge par un guide francophone. Des écouteurs sont distribués. Le guide et le témoin interviendront au micro. Nous quittons Auschwitz I à 12 h. La visite du matin a duré 3 h 15 . Nous déjeunons entre 12 h 15 et 13 h 30 .

Nous reprenons le car pour Auschwitz II - Birkenau à 13 h 30. Le guide commence son commentaire devant l'entrée principale à $13 \mathrm{~h} 45$. Nous découvrons les baraques de quarantaine. Nous longeons la Bahnrampe, puis nous entrons dans une baraque de détenus avant d'arpenter les ruines des Crématoires II et III plongées sous la neige. Après la visite du Zentral Zauna, nous retournons devant le monument officiel de 1967 pour une cérémonie à $16 \mathrm{~h} 5$. La nuit tombe. Il fait un froid polaire. La cérémonie se termine à $16 \mathrm{~h} 20$. Nous retournons au car en empruntant le chemin de la Bahnrampe. Nous sommes dans les cars à 16 h 50. Nous arrivons à l'aéroport de Cracovie à $17 \mathrm{~h} 45$. Nous décollons à $19 \mathrm{~h} 30$. Nous sommes de retour à Lyon à $21 \mathrm{~h} 40$.

\section{LA PÉRIODE HIVERNALE}

La neige est un obstacle pour la lecture historique du site. Quand on peut voir les ruines, notamment celles des structures d'extermination, une analyse archéologique, appuyée par des documents historiques et des témoignages, permet de construire une séquence pédagogique afin de lire le lieu. Par exemple, il est possible de croiser les données archéologiques des crématoires et des bunkers de Birkenau avec des dessins de David Olère, des documents de la Direction centrale des Constructions et de la firme Topf et des témoignages de Sonderkommandos, comme celui de Philip Müller dans Shoah de Lanzmann ${ }^{10}$. Mais avec la neige, nous devenons aveugles. D'autre part, l'hiver n'apporte rien à la connaissance d'Auschwitz. Les 400000 Juifs de Hongrie ont été assassinés au cours du printemps et de l'été 1944. L'industrie de la mort fonctionnait en toutes saisons. Et pour ceux, une minorité, sélectionnés pour la partie concentrationnaire, les étés étaient tout autant insupportables et meurtriers que les hivers. La saison ne nous apprend rien sur le processus d'extermination des Juifs. Sans compter que le froid gêne l'attention des élèves et que la nuit tombe plus vite, réduisant ainsi l'amplitude du temps de visite. Dans la mesure du possible, l'hiver est une saison à éviter.

\section{LA PRÉSENCE DES TÉMOINS}

Si le témoin est une rencontre inoubliable et indispensable pour les élèves, sa présence peut brouiller la spécificité du lieu. Les survivants d'Auschwitz sont majoritairement les témoins de l'univers concentrationnaire. Ils n'ont pas été les

(10) David Olère, L'CEil du témoin, New-York, The Beate Klarsfeld Foundation, 1989 ; Jean-Claude Pressac, Auschwitz. Technique and Operations of the Gas Chambers, New York, The Beate Klarsfeld Foundation, 1989, Les Crématoires d'Auschwitz, la machinerie du meurtre de masse, Paris, CNRS, 1993 ; Techniker der « Endlösung » Topf \& Söhne, die ofenbauer von Auschwitz, Stiftung Gedenkstätten Buchenwald und Mittelbau-Dora, 2005; Claude Lanzmann, Shoah, Paris, Gallimard, coll. «Folio», 1997. 
témoins oculaires de l'extermination, à l'exception des Sonderkommandos. Claude Lanzmann l'avait bien compris dans son film Shoah. L'histoire de la concentration n'est pas celle de l'extermination. Lors d'une visite, c'est la parole du témoin qui va retenir toute l'attention des élèves. Ainsi la spécificité du lieu, centre de mise à mort, s'efface. Au cours d'une visite avec un témoin, la stupéfaction des élèves ne naît pas de l'anéantissement de masses humaines à Birkenau, mais leur question est de savoir comment le témoin a pu survivre dans des conditions de vie aussi inhumaines. Pour les élèves, la question centrale ne devrait pas être « Comment survit-on à Auschwitz? », mais plutôt «Comment meurt-on à Auschwitz? »Cependant, si la visite avec le témoin est centrée sur le volet concentrationnaire, le dispositif pédagogique peut garder sa cohérence, à condition de bien insister sur la différence fondamentale entre le monde de la concentration et le monde de l'extermination et de disposer d'un temps raisonnable pour articuler la visite entre ces deux mondes ${ }^{11}$.

\section{LA QUESTION DE LÂGE}

Des réserves sérieuses sont émises pour les voyages d'élèves de Troisième en raison de leur jeune âge (entre treize et quinze ans). Il s'agit d'une période compliquée où les écarts de maturité dans une même classe sont souvent importants. L'émotion est parfois difficile à contrôler. Un simple effet de sidération peut bloquer le processus d'apprentissage. La question de la compréhension intellectuelle doit être posée. Ces élèves ont-ils une capacité d'abstraction suffisante pour comprendre ce qu'ils verront? À Auschwitz, la capacité d'imagination ne naît pas avec les objets ou le paysage, mais avec la connaissance et la réflexion. Est-ce le bon temps pédagogique ? Ce type de visite est sans doute prématuré pour des élèves aussi jeunes ${ }^{12}$. Aussi, est-il plus raisonnable de différer les voyages plus tard dans le temps scolaire, vers 16-18 ans, quand l'élève a plus de maturité, de connaissance et de recul. Une lettre de la Direction générale de l'enseignement scolaire, datée du 16 juin 2010, adressée aux Recteurs pour être diffusée auprès des corps d'inspection, préconise : « Pour les écoles élémentaires, les visites et les études de lieux de mémoire locaux [...] ; pour les collèges, les visites de sites locaux et nationaux plutôt que les voyages à l'étranger ; pour les lycées, les voyages locaux, nationaux et européens. » Ces préconisations ont été pensées dans le cadre d'une commission bilatérale entre le ministère de l'Éducation nationale et le ministère de la Défense. Cette réflexion a été « menée tant pour éviter les redondances

(11) Je garde le souvenir de deux voyages à Auschwitz où ce dispositif pédagogique a été respecté. Le premier, en mars 2006, avec Ida Grinspan, et 24 élèves de Troisième du Collège André Malraux de Paron, conduits par leur professeur de français, Vincent Moissenet. Avec Ida, nous nous sommes rendus sur le site de l'ancienne usine de l'Union- Werke dans laquelle elle avait travaillé. Le second voyage, à la Toussaint 2011, avec Paul Sobol et les jeunes du Centre Communautaire Laïc Juif de Bruxelles.

(12) Cf. les deux contributions de Pierre-Jérôme Biscarat : Les Voyages pédagogiques en Pologne des élèves de Troisième, rapport d'évaluation, Paris, Fondation pour la Mémoire de la Shoah, juin 2010 ; «Y a-t-il un âge pour aller à Auschwitz? », in Jacques et Ygal Fijalkow, Les Élèves face à la Shoah, op. cit., p. 64-80. Après quatre expériences de voyages à Auschwitz avec des classes de Troisième, entre 2005 et 2007, j’ai décidé de ne plus accompagner des élèves de ce niveau. 
dans le parcours des élèves que pour ne pas mettre des élèves trop jeunes face à des réalités traumatisantes ${ }^{13}$.»

\section{LA QUESTION DU NOMBRE D'ÉLÈVES}

Les voyages de masse où les élèves se retrouvent plusieurs dizaines, voire plusieurs centaines, ne me semblent pas compatibles avec des objectifs pédagogiques sérieux. L'effet de groupe génère de l'agitation et des difficultés pour les enseignants à la canaliser. Les élèves ont plus de difficultés à se concentrer. Un nombre compris entre vingt et quarante élèves me paraît plus raisonnable.

\section{LE POURQUOI ?}

Pourquoi se rend-on à Auschwitz ? Pourquoi emmener des élèves à Auschwitz ? Une des dérives est celle du « voyage symbolique » : Auschwitz présenté comme symbole quasi unique de la Shoah, Auschwitz symbole du mal absolu, Auschwitz comme outil symbolique pour vacciner les élèves contre le racisme et l'antisémitisme, Auschwitz comme outil pour encourager les élèves à s'engager pour la promotion et la défense des droits de l'homme, Auschwitz pour responsabiliser l'homme face à la barbarie, Auschwitz pour dire « Plus jamais ça ». Oui, bien sûr, il faut aborder avec les élèves les grandes questions intellectuelles, philosophiques, anthropologiques, morales, civiques soulevées par Auschwitz. Elles sont essentielles dans la démarche éducative. Mais toutes ces questions peuvent être traitées, en amont ou en aval de la visite des lieux, dans des salles de cours ou dans des temps pédagogiques spécifiques.

Lorganisation des voyages nécessite une logistique très lourde. Il faut monter les dossiers de subventions, demander les autorisations de sorties, convaincre son chef d'établissement, le Conseil d'administration, ses collègues, puis rassurer les parents. Avant le départ, il faut s'assurer que chaque élève a bien respecté les consignes administratives. De Lyon, le voyage en car pour Auschwitz est de 1500 km, soit environ vingt à vingt-cinq heures de trajet. Après tant d'efforts, autant profiter du lieu pour ce qu'il est. Mon parti-pris est de considérer Auschwitz comme un site strictement historique. La Shoah est un fait d'histoire ${ }^{14}$. Auschwitz est un lieu d'histoire. Les voyages pédagogiques ont pour vocation d'être des « voyages historiques ». Les faits, rien que les faits. Il est de notre responsabilité d'enseigner que les fondements d'un monde commun n'existent que s'il y a des vérités factuelles que l'on ne peut pas changer à volonté. Pour contrer le négationnisme et le relativisme, il est impératif de préserver la distinction entre les faits et les opinions. Je considère le lieu comme l'un des outils pédagogiques pour mieux apprendre l'histoire. Ce choix entend que l'on peut

(13) Pour donner suite aux conclusions du séminaire de l'ENS Lyon de mai 2011, la Fondation pour la Mémoire de la Shoah indique désormais que les « classes de collège (niveau Troisième exclusivement) peuvent également bénéficier des aides de la Fondation », mais uniquement « pour des visites de lieux de mémoire régionaux » (dossier « Voyages 2011-2012»).

(14) Georges Bensoussan, « la Shoah, fait d'histoire », Le Monde, 7 juillet 2000, p. 15. 
faire de l'histoire à partir du lieu, faire de l'histoire en arpentant les lieux, pas à pas.

Il y a une force des lieux. Le directeur du musée d'Auschwitz, Piotr Cywinski, déclarait: «Des cours sur la période du nazisme, sur la Shoah, on peut les faire partout. Si les gens viennent ici, c'est pour voir, pour imaginer, pour agrandir leur conscience de la réalité ${ }^{15}$. » Face à la force des lieux, seule une approche factuelle permet de tenir une distance et de susciter ensuite une intense réflexion. Le site d'Auschwitz a besoin d'histoire. Il ne peut pas supporter les approximations, voire les inventions. Quand vous êtes en situation, sur le site, avec trente élèves devant des lieux vides (des ruines, une prairie, une mare), vous avez besoin de faits. Quelle histoire pour les premières chambres à gaz provisoires, les Bunkers I et II ? Quelle histoire pour les Kommandos de Budy ${ }^{16}$ ou de Bobrek? Un lieu, des dates, une histoire. Nous avons besoin d'histoire, de faire de l'histoire, une histoire factuelle des lieux.

Les visites scolaires idéales n'existent pas, le public idéal non plus. Cependant, nous devons nous efforcer de faire au mieux. Pour s'assurer de la réussite d'un « voyage d'histoire », il faudrait sans doute moins d'élèves (le groupe Classe est adapté) et plus de préparation en amont et de réflexions en aval. Lapproche pluridisciplinaire doit être privilégiée. Le format d'une seule journée ou d'une demi-journée de visite me paraît difficilement compatible pour une bonne compréhension des sites du camp d'Auschwitz. Deux jours de visites sont raisonnablement nécessaires. De plus, la visite d'une journée prive les élèves de la visite des traces juives de Cracovie. Par son patrimoine, la capitale des rois de Pologne offre un condensé du judaïsme d'Europe de l'Est dans son absolue diversité. Il faut visiter Cracovie et ne pas se limiter au seul musée d'Auschwitz. Un format de visite se situant entre trois jours et une semaine permet d'inclure à la fois les traces juives de Cracovie et les lieux principaux d'Auschwitz ${ }^{17}$.

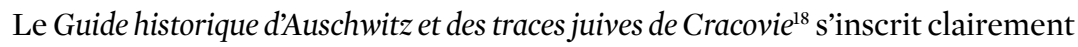
dans cette optique. Le texte précis et méticuleux de Jean-François Forges permet de rendre le site d'Auschwitz à l'histoire, puisqu'elle seule peut en assurer la lisibilité. Dans les lieux de mémoire traumatique, la démarche éducative doit avoir pour finalité de

(15) Piotr Cywinski, « Auschwitz, site mémoriel au XXIe siècle : réalités, enjeux, questions », in Annette Wieviorka, Piotr Cywinski (dir.), Le Futur d'Auschwitz, Actes de la journée d'étude du 11 mai 2010 à l'Université Paris 1 Panthéon-Sorbonne, Paris, Les Cahiers Irice, 2011, p. 9-25, cit. p. 13.

(16) Le Kommando agricole de Budy est l'un des lieux de la mémoire juive française d'Auschwitz. Au milieu des années 2000, les habitants du village ont fait apposer une plaque sur l'école, transformée en ferme à l'époque nazie : «Dans ce bâtiment se trouvait une compagnie disciplinaire du KL d'Auschwitz de prisonnières juives polonaises et de différentes nationalités. En octobre 1942, les gardiens SS et les prisonnières allemandes de fonction assassinèrent sauvagement 90 prisonnières, surtout des juives françaises. Honneur à leur mémoire ! » Ces juives françaises étaient de jeunes intellectuelles, étudiantes de la Sorbonne et artistes. En 2009, le musée d'Auschwitz a installé un panneau historique.

(17) Sur un format minimal de trois jours, l'expérience du programme expérimental conduit par le Département de la Vienne et le Mémorial de la Shoah, du 29 au 31 mars 2007, pourrait servir d'exemple. Le projet concernait deux collèges et s'articulait selon les axes suivant : « Liaison avec les programmes disciplinaires en histoire-géographie, en lettres, en arts plastiques ou en langues; les élèves découvrent la culture juive de Pologne et d'Europe centrale avant la Shoah : shtetl, ghettos, langue... ; ils abordent l'histoire de Cracovie et de la Pologne ; ils visitent Auschwitz avec des survivants de la Shoah. »

(18) Jean-François Forges, Pierre-Jérôme Biscarat, Guide historique du camp d'Auschwitz et des traces juives de Cracovie, préface de Piotr Cywinski, Paris, Autrement/ministère de la Défense, 2011. 
susciter une réflexion personnelle des élèves, une mise en perspective de l'histoire, un regard critique et lucide sur l'événement. Dans cet esprit, je reste réservé sur les bienfaits supposés d'une pédagogie du choc et des jeux de l'émotion, car la pédagogie n'a pas pour vocation de choquer, mais d'élever ${ }^{19}$.

(19) Entretien avec Philippe Quintin, professeur d'Histoire, membre du service pédagogique de la Maison d'Izieu (Izieu, 21 mai 2010). 